\title{
A Least-Cost Approach to Personal Exposure Reduction
}

\author{
Gemma Davies \\ Lancaster Environment Centre \\ Lancaster University
}

\author{
Duncan Whyatt \\ Lancaster Environment Centre \\ Lancaster University
}

Keywords: MasterMap, air pollution, journey-time exposure, space-time

Address for correspondence: Gemma Davies, Division of Geography, Lancaster Environment Centre, Lancaster University, Lancaster, LA1 4YQ. E-mail: gemma.davies@lancaster.ac.uk

\begin{abstract}
Concerns over the potential negative health effects from exposure to air pollution have led to interest in assessing personal exposure and finding ways to reduce it. As journey-time exposure accounts for a disproportionately high amount of an individual's total exposure, this paper assesses the potential to apply least-cost techniques within a GIS in order to identify paths of lower journey-time exposure.

The methodology adopted uses pollution surfaces for $\mathrm{PM}_{10}$ and $\mathrm{CO}$ generated by the dispersion model ADMS, with an analysis mask derived from OS MasterMap to create a least-cost surface. Actual routes taken by a cohort of 11-13 year old children on their journeys to school are used to compare observed journey time exposure with the exposure along alternative routes generated using the least-cost path function.

While the least-cost approach proved to be successful in defining low exposure routes the ability to scale up this approach is constrained by the amount of editing required to successfully create an analysis mask from OS MasterMap data. Such alternative routes have the potential to assist in promoting safer environmental choices, however, their likelihood of adoption is dependant on a number of social and environmental influences which affect an individual's route choice.
\end{abstract}




\section{Introduction}

Over recent years GIS has increasingly been used as a tool to assist with the modelling of air pollution and the analysis of exposure on an individual and aggregate basis. Its contribution includes the capture of information relating to pollutant emission and ambient concentrations and the subsequent integration of these concentrations with information about the population potentially at risk (Briggs 2005). Most studies are based on $\mathrm{NO}_{2}$ and $\mathrm{PM}_{10}$ as these pollutants are strongly associated with the road transport sector in urban environments. Around $70 \%$ of particulates in urban areas are accounted for by traffic emissions and road dust (Harrison et al. 1997).

Interest in improving methods for the assessment of personal exposure to airborne pollutants is stimulated by concerns over their potential negative effects on human health (Gulliver and Briggs 2004, Maynard et al. 2007). Evidence suggests a number of negative health outcomes including mortality, respiratory problems, allergies such as asthma, cardiovascular problems and cancer. In particular transport-related air pollution is associated with an increased risk of death from cardiopulmonary causes and non-allergic respiratory symptoms and disease (Heinrich et al 2005, RCEP 2007). Researchers have concluded that both short-term and long-term variations in pollutant concentrations have adverse effects on health (RCEP 2007), with many chronic effects simply the result of repeated short-term exposure to elevated concentrations of pollutants (Gulliver and Briggs 2004). Walking in heavily trafficked environments significantly increases exposure, with levels of exposure being potentially exacerbated by traffic, weather, and hotspots (Greaves et al 2008).

Whilst fixed-site automatic monitoring stations have been used as a measure of ambient air pollution in assessments of personal exposure at the population level, they exist in insufficient numbers to capture significant gradients in urban micro-environments and are not therefore suitable for detailed personal exposure assessment (Briggs 2005). Gauvin et al (2002) confirm in their conclusions that short-term personal exposure is not closely related to ambient air concentrations and is not therefore suitable for assessing population exposures. Alternative methods of deriving concentrations are therefore required. More detailed estimates of personal exposure can either be derived directly through mobile personal monitoring devices, or indirectly through a modelling approach which simulates the dispersion of pollutants based on information from an emissions inventory. Representations of individuals movements in time and space also need to accompany modelled concentrations in order to derive personal exposure estimates.

\subsection{Monitoring}

Portable personal monitoring devices may be used to directly assess exposure to particulates and other pollutants in a variety of micro-environments (Chan et al. 2002, Gauvin et al. 2002, Zhao et al. 2004, Kaur et al. 2005, Briggs et al. 2008). For example, Greaves used portable aerosol monitors to monitor exposure to $\mathrm{PM}_{2.5}$ along heavily and lightly trafficked streets in Sydney Australia. Monitored data was accompanied by supplementary spatial and contextual information recorded using a Global Positioning 
System (GPS) and voice recorder (Greaves et al. 2007). The VESTA study, France, also used active samplers for personal monitoring. These were carried by participating school children in 5 different cities to monitor their personal exposure to $\mathrm{PM}_{2.5}$ over a 48 hour period (Gauvin et al. 2002). While portable monitoring devices are probably the most direct and reliable means of assessing personal exposure, especially when accompanied with detailed route information using a GPS, the equipment is costly, hence sample sizes tend to be small. Furthermore, use is restricted to an assessment of present day pollution concentrations. Modelling approaches in contrast offer more flexibility.

\subsection{Modelling}

An alternative to the use of personal monitoring equipment is to model the distribution of pollutant concentrations using a spatially resolved emission inventory, which can then be integrated with positional information representing routes taken by individuals. Emissions are derived from information in the inventory about the traffic flow, composition and speed relating to each road source specified. These may be based either on traffic counts collected by local authorities (Jensen et al. 2001) or by using Origin-Destination matrices to develop traffic flow models (Armstrong and Khan 2004). Inventories can also contain nontraffic related emissions, such as significant industrial sources.

Once an inventory has been created various methods may be used to model the distribution of pollutants across geographical space. The simplest approach uses GIS techniques to create buffer zones of potential pollution based on distance from road and general traffic volumes (English et al. 1999, Gordian et al. 2006, Zandbergen and Green 2007). A slightly more sophisticated approach to modelling emission rates can be developed by incorporating variables such as travel speeds, and fleet composition for sections of road within an inventory (Armstrong and Khan 2004, Borrego et al. 2006). Further improvements in representing spatial variability in the distribution of pollution concentrations can be achieved through the application of dispersion models. Dispersion models take into account the spatial and temporal distribution of emissions, along with meteorological parameters such as wind speed, direction and temperature, and use these to generate surfaces representing pollutant concentration and deposition values (Colvile and Briggs 2000). The Space-Time Exposure Monitoring System (STEMS) developed by Gulliver and Briggs (2004), uses information from a traffic flow model, along with meteorological data as inputs to a dispersion model, ADMS. These surfaces are combined with data from time-space activity surveys in order to estimate exposure for individuals in time and space. For more information regarding the range of modelling approaches which can be applied to exposure assessment see Briggs (2005) and Jerrett et al (2005).

\subsection{Exposure Assessment}

Methods for assessing personal exposure to airborne pollutants have often focused on pollutant concentrations recorded or modelled at an individual's residential address, school or workplace (English et al. 1999, Heinrich et al. 2008). With the help of GIS it is feasible to develop increasingly realistic models of exposure taking into account time and activity patterns of the population (Briggs 2005). In Denmark, for example, a national system has 
been developed to support air quality management for large cities. The approach adopted here was to model exposure based on time spent at home, at work and in the streets, using estimates of the time spent by individuals in each environment and the indoor-outdoor exposure ratios for different environments (Jensen et al. 2001). A population-wide analysis of exposure using modelled air pollution and statistically derived time-activity patterns has also been carried out in Lisbon, Portugal (Borrego et al. 2006). Crabbe et al (2000) use GIS to combine a mixture of monitored concentration data, modelled concentration data and self-reported time and place specific information in order to assess the effect of the environment on health. Space-time activity surveys, however, fail to account in any detail for varying levels of exposure while travelling between locations.

Although relatively little of someone's time is spent travelling, journey-time exposure contributes a disproportionately high percentage of total exposure to air pollution, consequently having potentially important implications for health (Gulliver and Briggs 2005), with evidence suggesting that short term peak exposures of less than an hour may be significant to health (Michaels and Kleinman 2000). Gulliver and Briggs (2005) applied their STEMS model to generate estimates of journey-time exposure for a sample of 50 children travelling to school in Northampton, UK. As details of the individual travel routes were not available, home and school addresses were used to derive surrogate route information using ArcInfo's PATH function, however, evidence suggests that in reality the choice of route used for the school journey is more complex than this (Duncan and Mummery 2007, Bamford et al. 2008, Walker et al. 2009). The use of derived route information in journey-time assessment is therefore likely to over-generalise both the route taken and the speed of travel.

\subsection{Reducing Journey-time Exposure}

The choice of route taken by individuals in travelling between locations can influence both the concentration and duration of exposure they experience (Kaur et al. 2007). Where intervention studies have taken place to reduce exposure to transport-related air pollution it has been shown that such reductions may directly reduce health effects such as acute asthma (Heinrich et al 2005). Heterogeneity in urban air quality is seen within individual cities as well as between them, which is reflected by the variable range of exposure experienced along different routes with a localised area (Kaur et al. 2007). Kaur et al (2005) observed noticeable variation in pollution concentrations between two sides of the same street in central London, UK, thus suggesting potential value in identifying routes of lower exposure, even where the variation in route may appear quite subtle. Even greater reductions in exposure are observed when quieter side streets are used in preference to busy main roads (Greaves et al. 2008).

Hertal et al (2008) have sought to derive routes representing least journey-time exposure using a GIS route-finder approach. They adopted annual diurnal traffic (ADT) as a proxy for pollution exposure along given segments of a route network, based on the assumption that CO and NOx are highly correlated with ADT. This approach, however, fails to account for the increased cumulative exposure which results from longer journey times. In the case of $\mathrm{NO}_{2}$ and $\mathrm{PM}_{10}$, where background concentrations have a stronger influence than they have for NOx and $\mathrm{CO}$, some low exposure routes saw only marginal reductions or 
even slight increases in total exposure (Hertel et al. 2008). This paper seeks to derive routes of lower exposure, using a least-cost path approach, that will take into account both pollution concentrations and distance, thus identifying routes able to minimise journey-time exposure.

This paper explores the potential to identify routes for the school journey with lower journey-time exposure than those currently used. The case study focuses on a group of eleven 12-13 year old pupils from an urban secondary school in the north of England. This group was selected from a group of 34 children participating in a wider study designed to explore the use of mobile phone technology in assessing the impact of traffic related air pollution on children during their school journey (Bamford et al. 2008, Walker et al. 2009, Whyatt et al. 2008). This sample group were selected on the basis that they either walked or cycled to school, as the least-cost surface modelled in this study utilised open spaces not traversable by car or bus. Children walking or cycling also have potentially greater autonomy over their choice of route. Inclusion in the study was also dependant on good quality observed route data being available for the child, against which modelled routes could be compared. Detailed information about actual route choice was recorded at 1second intervals using a GPS. The focus of this paper is to assess the feasibility and usefulness of using least-cost path analysis within a GIS to define routes of least exposure to traffic related air pollution, which may be compared against journey-time exposure for the existing 'observed' routes. The exposure analysis will focus on $\mathrm{PM}_{10}$ and $\mathrm{CO}$.

\subsection{Least-cost applications}

The generation of least-cost paths within a GIS enables routes to be calculated which take both Euclidean distance and a weighting from a cost surface into account (DeMers 2005). In order to find a minimum path across a surface it is necessary to define an accumulated cost surface from a departure point which takes into account this cost value (Collischonn and Pilar 2000). A least-cost path is then traced through the accumulated cost surface to a destination point, in order to generate the least-cost path (Atkinson et al. 2005). Cost surfaces can be used to generate least-cost paths between any set of points within an area (Rothley 2005). Common examples of least-cost analysis include analysis of habitat connectivity and animal movement (Ganskopp et al. 2000, Halpin and Bunn 2000, Gonzales and Gergel 2007) and the use of least-cost surfaces in the planning routes for new transport infrastructures (Collischonn and Pilar 2000, Arima et al. 2005, Atkinson et al. 2005), however, there are currently no examples of this approach being applied in the context of exposure reduction.

\section{Methodology}

In order to create a least-cost surface and subsequent least-cost path information, a cost surface and origin and destination points are required. The inputs and method used to derive least-cost assessments of journey-time exposure are summarised in Figure 1. In this example of least-cost path analysis the cost surface relates to pollution concentrations derived from the dispersion model ADMS. The origin point is the location of the school 
and the destination points, the addresses of the participating school children. The final and perhaps most important element of the analysis is the analysis mask; essential in constraining journey movement to areas of publically traversable space.

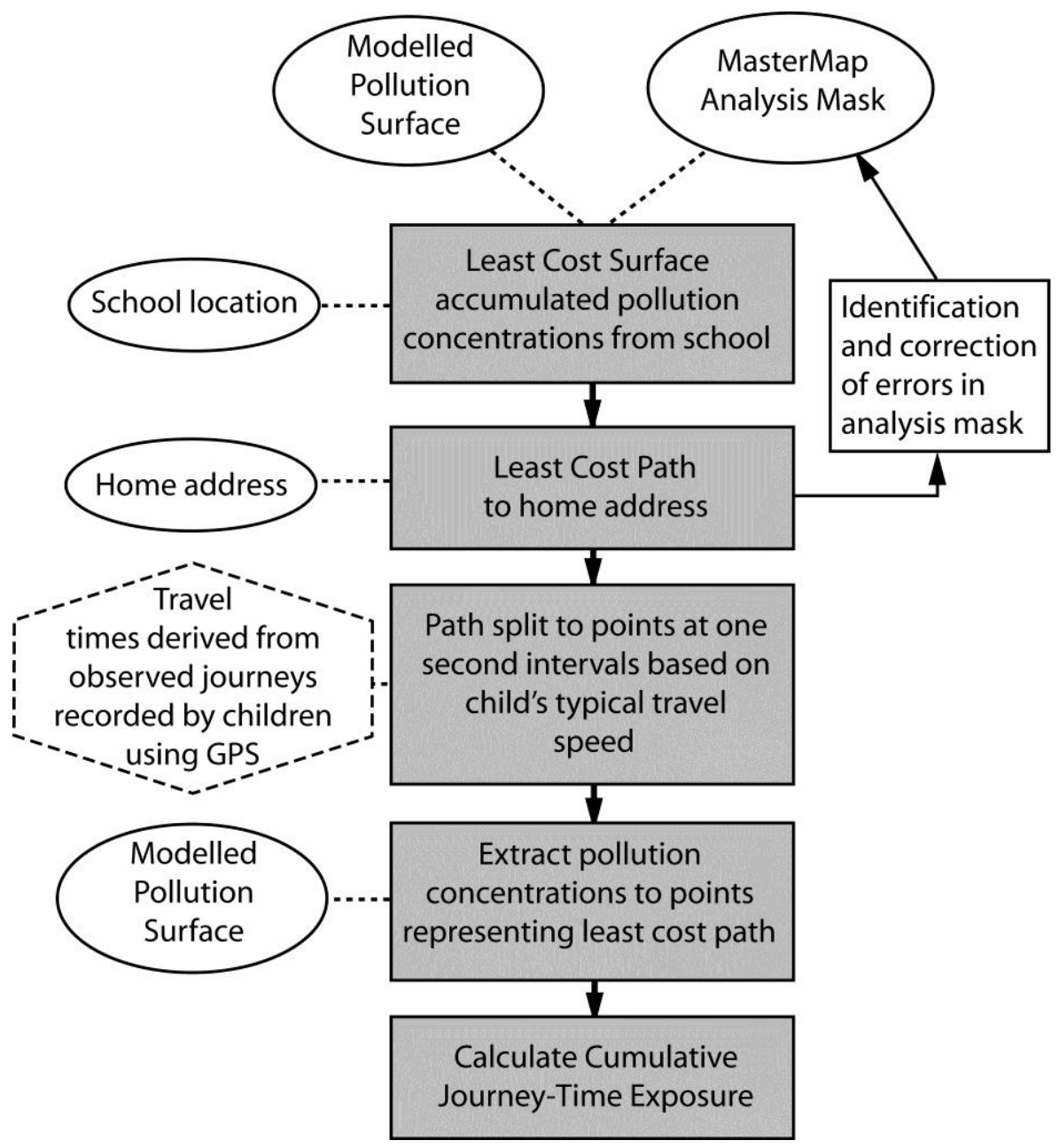

Figure 1: Summary of Methodology

\subsection{Pollution Surface}

Potentially any gridded representation of pollution could be used as the cost surface for the least-cost analysis. In the example presented here the dispersion model ADMS Urban (CERC, 2006) was used to generate estimates of traffic-related pollution for a range of pollutants including $\mathrm{PM}_{10}, \mathrm{NO}_{2}$ and $\mathrm{CO}$. Road geometries were captured from the road features in the OS MasterMap topography layer, with road widths also derived from this layer. A detailed emissions inventory for major roads in the study areas was constructed from traffic count and composition data supplied by the County Council and supplemented with estimates from the PARAMICS traffic simulation model. While fixed site monitoring data shows that pollution is far from static over time, a modelled approach is only able to 
generate average concentrations over time scales, for which required input data such as traffic flows are recorded. For the purpose of this study weekday traffic counts for the peak school journey period of 0815-0830 were used, as there was little variation in modelled output between this period and the surrounding time periods within which some of the children would be travelling to school.

The dispersion model incorporated background concentrations of air pollutants downloaded from the Local Authority section of the Air Quality Archive http://www.airquality.co.uk/archive/index.php and representative meteorology for a number of stations in NW England. The model could be run with any combination of meteorological conditions. The paper focuses on the use of meteorological data for a typical spring day, based on locally observed data, with a south-westerly wind direction as this is the predominant wind direction for the study area; however, the results are sensitive to variation in meteorology as will be demonstrated later. Model performance was verified against data from a dense network of $\mathrm{NO}_{2}$ diffusion tubes maintained by the City Council and additional sites chosen by the research team to capture other important locations in the study area (including the school playground and a nearby park). Model performance was also assessed relative to hourly $\mathrm{NO}_{2}$ and $\mathrm{PM}_{10}$ data captured at an automatic monitoring station located in the city centre.

ADMS Urban was run to a $30 \mathrm{~m}$ grid of receptor points across the study area, with the intelligent gridding option to place additional receptor points close to road sources. The model results were then interpolated using the ADMS-ArcGIS Link Toolbar in ArcGIS to create pollution-specific grids for $\mathrm{PM}_{10}$ and $\mathrm{CO}$ covering the study area at $0.5 \mathrm{~m}$ resolution.

\subsection{Origins and Destinations for Route}

The origin point for the least-cost surface was taken as a representative point outside the entrance to the main school building. The design of the subsequent analysis mask ensured that all of the school grounds and the entrances to both the north and south of the grounds were identified as traversable space. Destination points for the least-cost path were the home addresses of the school children. These were located using end points from observed route data collected with the GPS. Where children had two potential home addresses, the home they travelled to and from most often was used in this analysis.

\subsection{Creation of Analysis Mask (MasterMap)}

Having derived a spatially-variable pollution surface the next stage of the analysis was to develop an 'analysis mask' to constrain the movement of individuals to geographical spaces that were freely traversable. The mask, therefore, needs to a) define areas of publically traversable space, whilst b) taking into account physical barriers to movement such as walls. The mask is primarily based on the Ordnance Survey's MasterMap topography layer, which represents objects in the physical environment such as buildings, fields and fences as a combination of point, line and polygon features at a spatial scale of approximately 1:1,250. The high level of spatial detail of this product combined with its 
comprehensive feature attribution make it an ideal base for the creation of an analysis mask for detailed local studies such as this.
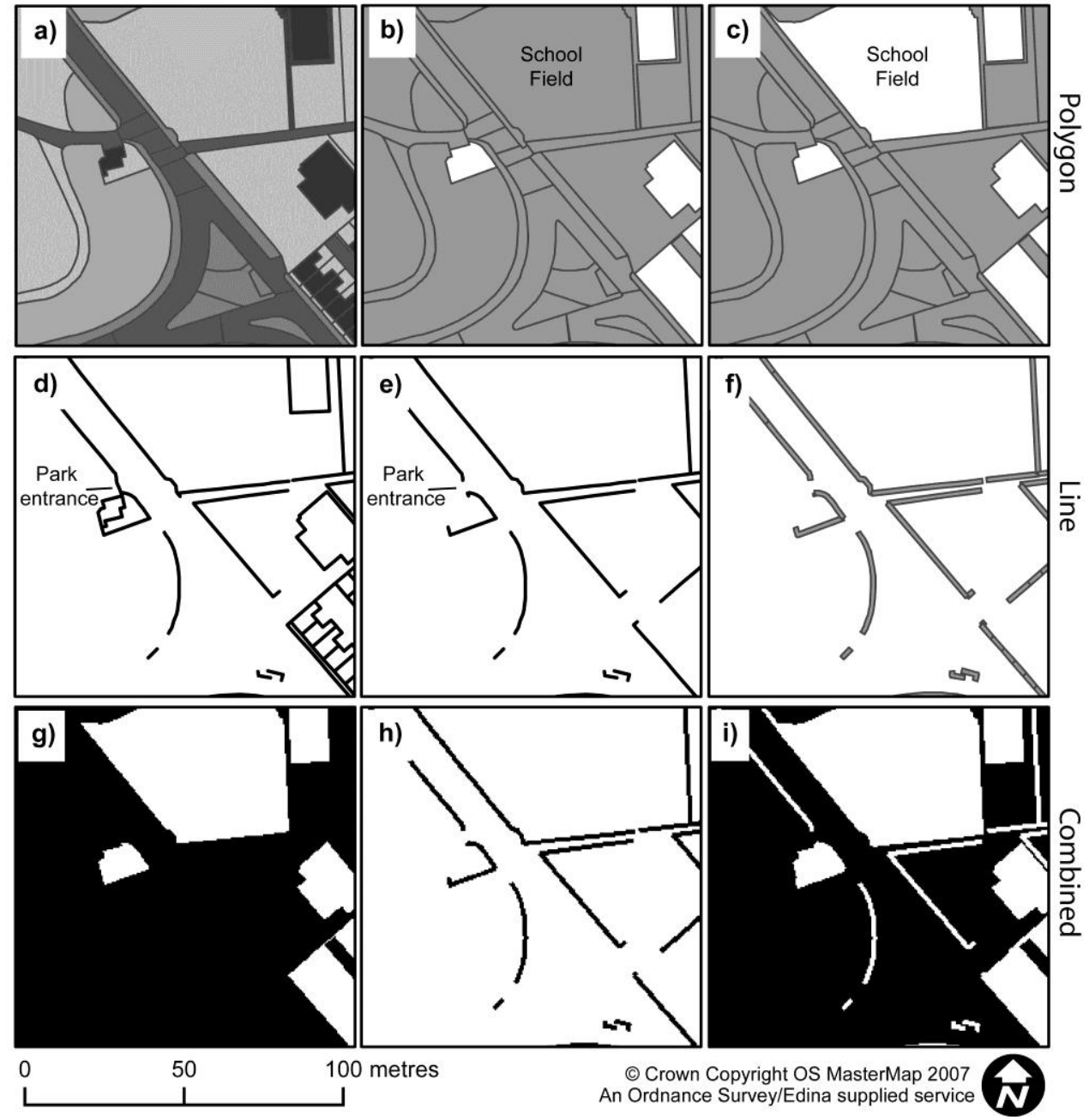

Figure 2: Use of Ordnance Survey MasterMap data for creation of an analysis mask

Inputs and processes involved in the creation of the analysis mask are summarised in Figure 2. The initial step in the creation of a mask was to select, based on attributes, those features that are clearly non-traversable, such as water, buildings, and railway, then to remove these from the layer of MasterMap polygons. It was found, however, that selection based on feature attributes alone created some gaps in the data set, for example where the data represents a none traversable railway, which in the real world crosses over a traversable road, therefore leaving a gap in the road network which needed to be filled. Selecting features based on attributes alone left a variety of other features represented as potentially traversable which would not be accessible to members of the general public. Examples include a local sewage works and school playing fields. These features, therefore, needed to be removed after manual selection using information from other sources, such as 
Ordnance Survey 1:10,000 maps, the MasterMap annotation layer and local knowledge. Figure 2c shows a school playing field now removed from the features selected as traversable within the mask.

The next stage is to incorporate into the mask features represented as lines which act as barriers to movement, and which are not already accounted for by the polygon features from the previous step, such as walls and fences. Linear features that were considered to be barriers to movement were initially selected as those features attributed as having an 'obstructing' physical presence. Figure $2 \mathrm{~d}$ depicts all line features attributed with a physical presence. The Ordnance Survey definition of physical presence is as follows: "The physical presence attribute indicates the real-world presence of the object represented by the feature. For example, a value of obstructing indicates that the feature prevents pedestrian access, whereas a value of edge/limit means that the feature represents a change of surface type and does not generally impede access" (Ordnance Survey 2007: pg 44). Relying only on the attribute for physical presence would, however, generate false barriers to movement. For example gates are represented as having a physical presence; yet do not create a barrier to movement. Likewise barriers such as walls and fences alongside overhead features such as bridges may create an apparent barrier at ground level that does not actually cause an obstruction to movement. Figure $2 \mathrm{e}$ shows those line features remaining after the removal of features identified as traversable, for example a gateway into the park and those features already accounted for by polygon features.

Both the polygon and line features were converted to raster layers before being combined to create the final version of the mask (Figure $2 \mathrm{~g}$ and $\mathrm{h}$ ). A resolution of 0.5 metres was chosen for the mask and subsequent analysis, as at coarser resolutions the integrity of fine details such as gateways often little more than 1 metre wide would otherwise be lost. There is no advantage in applying a finer resolution for the mask or subsequent analysis in this case, as sub-metre variations in the interpolated pollution surface are negligible. Before the selected line barrier features could be converted into a raster format and subsequently subtracted from the polygon elements of the mask, a $0.75 \mathrm{~m}$ buffer was applied to the line features (Figure 2f). Without the application of buffering cracks may develop in the mask and subsequent least-cost surface, potentially producing unrealistic least-cost paths at a later stage in the analysis (Rothley 2005). Rothley (2005) goes on to explore further more sophisticated means of filling potential cracks in barriers, however, at the high resolution we chose to work with the simple buffering of line features before converting them to raster successfully avoided unwanted cracks in the barriers, whilst not compromising the validity of surrounding features. An example of part of the final analysis mask is shown in Figure $2 \mathrm{i}$, with traversable spaces shown in black.

\subsection{Least-cost Paths}

Using the MasterMap-derived mask to limit the extent of the least-cost surface, an accumulated cost-distance surface was generated using ArcGIS 9.2 (ESRI 2006). The least-cost surface produced represents an accumulated cost taking into consideration both Euclidean distances from the school studied and the pollution concentrations from the outputs modelled in ADMS. 
Once a least-cost surface has been developed, a least-cost line is traced across the accumulated cost-distance surface to given destination points (Atkinson et al. 2005), in this study these represent the home addresses of the school children.

\subsection{Journey-time Exposure}

The observed data which is used as a baseline against which to compare the least-cost routes, comprises of a series of points, collected by the pupils using a GPS during their school journey. This information was collected at 1-second time intervals on journeys both to and from school during four, week long study periods spread throughout 2007 (Bamford et al. 2008, Whyatt et al. 2008). The routes used in the analysis were those considered to be most representative of those taken between home and the school. It is, however, recognised that many children use a variety of different routes depending on personal circumstances, which vary according to day of the week, time of year, and the weather amongst other factors. Some children simply vary their choice of route as they prefer to have variation within their journey pattern (Walker et al. 2009).

Cumulative exposure was calculated by extracting values from the modelled pollution surfaces to points representing positions at 1-second intervals along the routes. In order to make comparisons between the observed route data collected and the least-cost routes generated, average journey speeds were calculated from the observed data for each child. These speeds were then applied to the least-cost paths in order to split them into an appropriate number of points to represent 1 -second intervals.

\section{Results}

Journey-time exposure based upon observed route data varies between children in relation to two main factors a) the time taken to complete their journey, and b) the mean pollution concentrations they are exposed to. Louise $^{1}$ for example, walks along a busy main road (Figure 3) and is, therefore, exposed to high pollutant concentrations which, combined with a relatively long journey period, mean she experiences a higher cumulative exposure than any of the other children (Table 1a). In contrast Katie whose journey takes 5 minutes longer than Louise's experiences less cumulative exposure as her route is predominantly through less polluted parkland.

The potential reductions in exposure to $\mathrm{PM}_{10}$ and $\mathrm{CO}$ which could be made by adopting least-cost paths for the school journeys in this case study are presented in Table 1. Within the cohort of 11 children who walked or cycled to school on a regular basis, potential reductions in $\mathrm{PM}_{10}$ exposure of up to $23.1 \%$ were observed, with an even greater potential to reduce $\mathrm{CO}$ exposure by up to $40.7 \%$. The greatest potential for reduction was observed for Louise's school journey. Here the observed route data show that she experiences both the highest concentrations as well as the highest cumulative exposure. This is explained by a choice of route along the upwind (predominant south-westerly) side of the busy main road, which is often congested during school journey-times. Figure 3 shows her observed

\footnotetext{
${ }^{1}$ Pseudonyms have been used when referring to the children participating in the study.
} 
route along with the suggested least-cost alternatives for $\mathrm{PM}_{10}$ and $\mathrm{CO}$. The least-cost $\mathrm{PM}_{10}$ route starts alongside the main road, and then uses a nearby cycle route which is offset from the main road, situated along the banks of a river. The least-cost $\mathrm{CO}$ route is similar, however, the earlier stages of the route divert through a housing estate before joining the cycle route. Neither route adds much time to the duration of her journey. The variation between least-cost routes for $\mathrm{PM}_{10}$ and $\mathrm{CO}$ is explained by the sharper decay in $\mathrm{CO}$ concentrations to background levels with distance from road.

a) Exposure to $\mathrm{PM}_{10}$

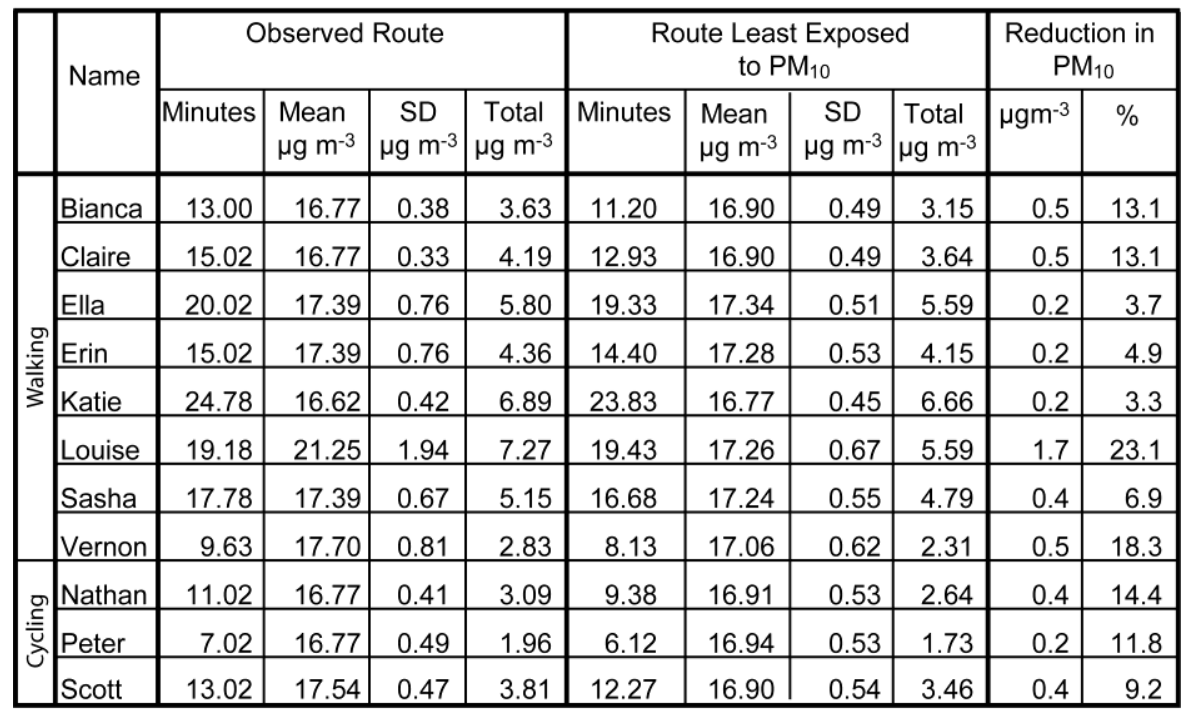

b) Exposure to $\mathrm{CO}$

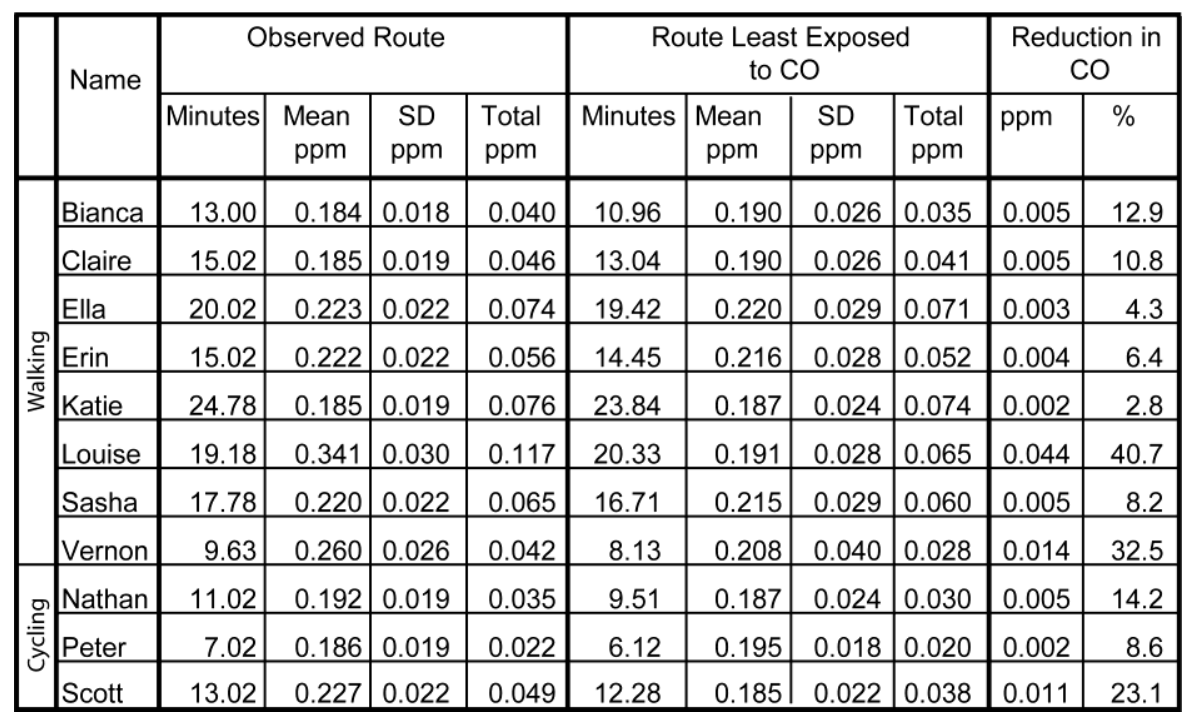

Table1: Potential reduction in journey-time exposure from the application of least-cost path analysis. 


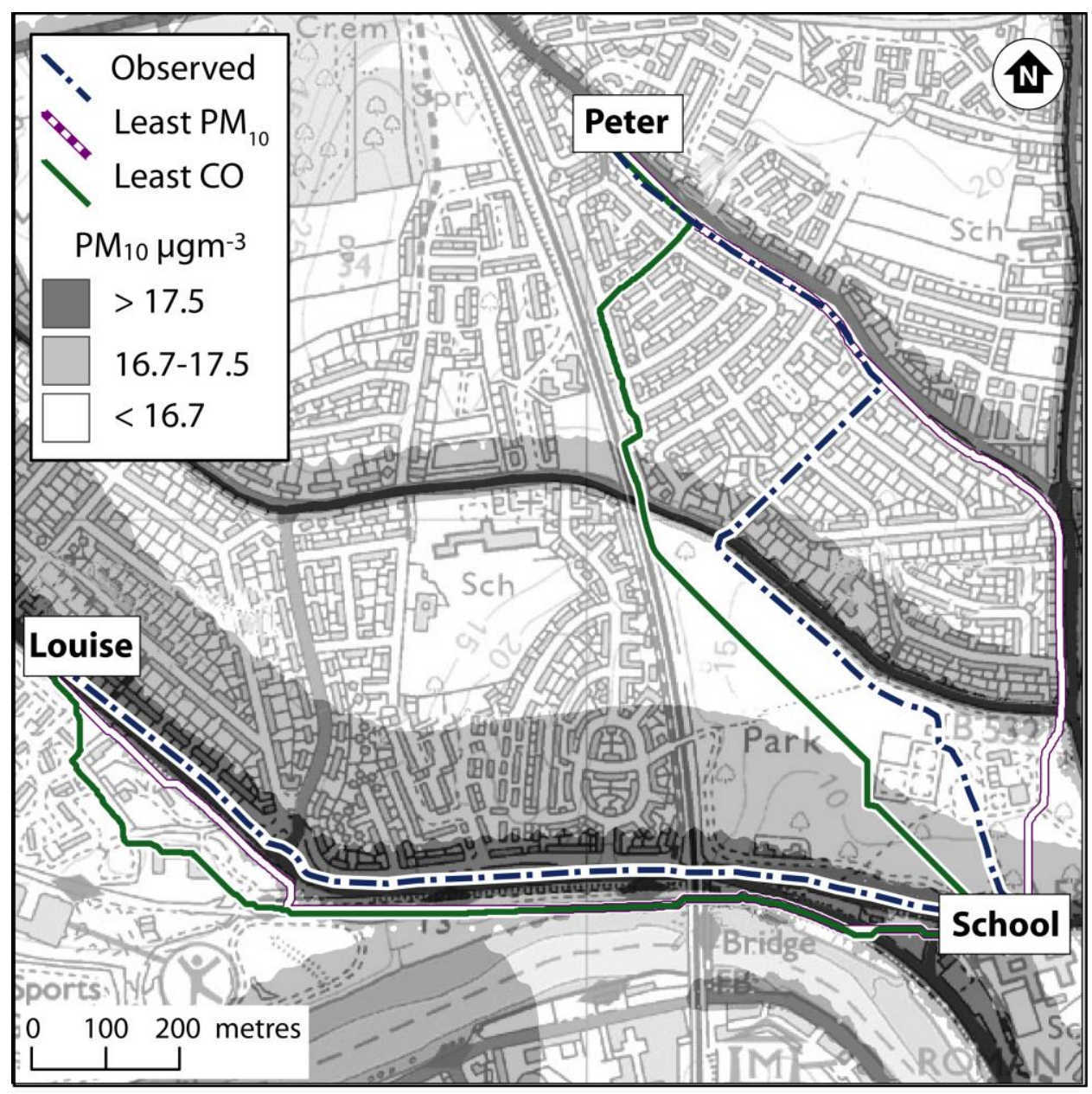

Based upon Crown Copyright OS 1:25,000 raster, 2007. An Ordnance Survey/Edina Supplied Service

Figure 3: Observed and least-cost routes for Louise and Peter, shown with pollution concentration for $\mathrm{PM}_{10}$

Greater spatial variation in route choice is observed for Peter (Figure 3). In his case both least-cost alternatives have slightly lower journey times than his observed route, with the least-cost $\mathrm{PM}_{10}$ route (Table 1a) minimising journey duration and the least-cost $\mathrm{CO}$ route Table $1 b)$ showing a greater offset from the main roads. For those children where there are no significant open spaces between home and school the spatial variation between leastcost paths for $\mathrm{PM}_{10}$ and $\mathrm{CO}$ is lower. The potential for reduction in journey-time exposure varied greatly between children. For some the route already taken was very close to the least-cost generated routes. Even in these cases, however, subtle changes in behaviour could still lead to a reduction in journey-time exposure. For example, by simply leaving it until the later stages of her journey before crossing the road from the west (upwind) to the east (downwind) side of the road Erin could see a reduction in exposure of $4.9 \%$ for $\mathrm{PM}_{10}$ and $6.4 \%$ for $\mathrm{CO}$. 


\section{Discussion}

One of the key advantages of using least-cost methods for identifying routes to reduce journey-time exposure is that the method offers the opportunity to incorporate pollution concentrations into route choice while also accounting for the length of journey and, therefore, exposure duration. Travel through a least-cost surface also has advantages over routes derived through a network driven approach, as subtleties such as side of the road or choice of route across open ground can be taken into account. As these subtle variations have potentially significant impacts on levels of exposure (Kaur et al. 2005, Kaur et al. 2007) the detail offered though this approach clearly facilitates the identification of less exposed routes. Working at a resolution of $0.5 \mathrm{~m}$ enables the effect of steep pollution gradients to be taken into account making the least-cost paths sensitive to which side of road is traversed. One element this approach to journey-time exposure fails to account for is short pause points, often at pollution 'hot-spots', such as the time spent at junctions waiting to cross a road. These pauses are represented in the observed route data, however, the least-cost routes assume constant travel speed, and therefore fail to account for pauses on route, which leads to a slight over-exaggeration of the benefit of low exposure routes. Furthermore, while the dispersion model in this approach takes into account terrain and the effect of urban canyons, it is acknowledged that any buffering effect from subtle barriers such as trees is not accounted for (Beckett et al. 2000).

The least-cost approach enables analysis to be applied to multiple people, multiple destinations and even future scenarios (e.g. increases or decreases in traffic flows) with relative ease and provides a flexible and cost effective alternative to exposure monitoring. Identification of least-cost pathways through the environment may be used to assist in promoting healthy walking routes, for instance the increasing use of walking school buses designed to promote healthy transport to school. The proportion of children either walking or cycling to school varies considerably between countries (UK 46\%, USA 13\%, Denmark 75\%) (Osborne 2008). There are also differences between urban and rural areas, with 50\% and $21 \%$ of children walking to school in the UK respectively (DFT 2007). While walking buses already have the advantage of reducing vehicle use (Kearns et al 2003), routes could be designed to minimise journey-time exposure, thus furthering their goal to promote healthy transportation. The design of less exposed alternatives for travel in urban areas may also assist with new urban design principles aimed at integrating health concerns onto the design and management of new urban systems (RCEP 2007), such as designing pedestrian routes separated from heavily trafficked routes and incorporating access to urban green space.

MasterMap topographic data provided a valuable basis upon which to develop the analysis mask for this study. The development of an appropriate mask at a suitably high spatial resolution has proved essential to meaningful development of least-cost analysis in this context. The introduction of easy and affordable access to MasterMap to the academic community through the Edina Digimap agreement has opened up new opportunities for researchers wishing to exploit this rich and detailed set of spatial information. Significant limitations in the extent to which appropriate features could be selected from the topographic layer in an easily automated manner were, however, encountered. This 
restricts the ease with which this approach could be adopted in larger study areas elsewhere. While it was possible to automate the majority of the editing process it should be noted that even small features missing from the mask, such as entrances to the park can have huge implications on the quality of any subsequent analysis. For small-scale studies, especially where cartographic information is accompanied by good local knowledge of study area, a careful iterative process to the development of a realistic analysis mask has been demonstrated to be successful. There is scope, however, to use more complex queries and algorithms to pull out features from MasterMap as Regnauld and Mackaness (2006) demonstrated in creating hydrographic networks from the cartographic feature representations provided in MasterMap. In this context MasterMap was the most useful detailed topographic data available, however, the same principles of selecting traversable features from which to create a mask could be applied to other data sources elsewhere in the world as long as sufficient detail is available from which to identify available pathways and potential barriers to movement.

While the mask created in this example was applied to both walkers and cyclists, variations in transport type may warrant different restrictions on travel movement. In this case study for example, the least-cost path identified for Scott uses a footpath alongside a railway bridge as his route over the river, however, Scott cycles to school and there is a flight of steps leading up to this bridge. This route while suitable for walkers is clearly less suited to those travelling by bicycle. By adjusting the mask to take into account the flight of steps, the least-cost path is rerouted via a foot bridge, and closely resembles the observed route that Scott already chooses to take. While feature-based approaches such as network analysis are able to take into account feature hierarchy, the raster based least-cost approach described here is only able to model the real world in 2-dimensions. Within this study it was not found that this had a significant bearing on the results derived.

While restrictions were placed on which land surfaces were classified as traversable such as the obvious need to avoid walking through water or walls, all grassed or wooded areas were classified as traversable. While this was necessary in order to allow grassed or wooded areas to be used as short cuts or links between other pathways, it fails to account for the reality that observed routes tend to favour the use of surfaced paths, especially if the ground is wet. Potential further work could incorporate a layer representing surface type into a weighted cost surface, to more closely reflect the realities of path choice and the potential variation in speed of travel across different surface types. While using a weighted multi-criteria cost surface may improve the extent to which potential routes reflect reality, further research is needed to ensure that any weightings applied are meaningful.

The approach adopted here uses meteorological data representative of a typical spring day in 2006. The wind direction used is south-westerly as this is the dominant wind direction in the study area. The model results and subsequent analysis are, however, sensitive to variations in meteorology, especially wind speed and direction. Exposure analysis was repeated for 'base case' (observed) and least-cost case holding all variables other than wind direction constant. Least cost routes determined with a change in wind direction vary at least slightly in all cases. This change is sometimes subtle, relating to a change in the side of road the least-cost path travels along, or in some cases is more significant, rerouting to the opposite side of parkland. Future potential applications of this method would benefit from a more dynamic treatment of meteorology allowing start and end locations to be 
inputted into a system, along with forecasted meteorological data for the time of the journey, in order to identify optimum routes relevant in both space and time.

Applying least-cost techniques in GIS to develop low exposure routes for the school journey or any other form of travel appears to show much potential, and fits the view of Colvile and others that even small adjustments to route choice such as avoiding time near kerbside when crossing busy roads can make significant differences (Guardian Unlimited, 2007). It is, however, important not to ignore other factors that influence route choice and may affect the likelihood of suggested least-cost routes being adopted. The observed data used in this case study to illustrate the 'typical' journey to school fail to demonstrate the range of variation in routes used by many of the children. Understanding of this variation in route choice was obtained via information in the form of photos and text messages captured using a customised mobile phone application and a series of follow up interviews with the participating children (Bamford et al. 2008, Walker et al. 2009). This element of the project revealed a diverse range of influences on route choice. These influences are varied and include: fear, parental control factors, the weather, meeting with friends, variations in family routine and presence/absence of day light (Walker et al. 2009). The importance of these factors relative to exposure to air pollution is likely to vary between individuals. For example, least-cost paths derived for Vernon could potentially reduce exposure to $\mathrm{PM}_{10}$ by up to $18.3 \%$ and $\mathrm{CO}$ by up to $32.5 \%$, however, the least-cost routes pass through the park near the school, in which he has previously experienced bullying and where his parents no longer allow him to walk. In cases such as this it is unlikely that concerns over exposure to air pollution will outweigh other factors. For some individuals the strength of these other factors is likely to be less significant and the option to make informed choices about alternative, potentially healthier routes is more likely to be embraced. Likewise where the variation in route is fairly minimal such as the suggestion to walk on the opposite side of the road, it may be expected that individuals would be more likely to adopt the alternative route. Potential exists to combine least-cost methods for exposure reduction with other elements such as street safety (Ellul and Calnan 2008).

The use of detailed observed route data as a baseline against which to compare least-cost alternatives has enhanced this study, not only by increasing understanding about route choice, but more relevantly by highlighting that the route taken is often not the shortest route and, therefore, not easily reproduced by network functions in a GIS. One of the key challenges of this approach is to communicate the benefits of exposure reduction to the general public in a timely and effective manner. Jenson et al (2001) developed a decisionsupport tool to assist local authorities in the management of air quality and personal exposure at home and workplace addresses. The exposure assessment and reduction techniques described in this paper could be developed in a similar manner. Alternatively, location-specific advice on low exposure routes could be streamed direct to a mobile phone providing individuals with the opportunity to make informed decisions in real-time. Such a service could be based on modelled estimates of air pollution, pre-calculated for a range of different wind speeds and directions, or based on real-time data captured by the mobile device itself. Nokia, for example, recently publicised their Eco Sensor Concept (Nokia 2007), comprising of a wearable sensor (capable of sensing and analysing an individual's health and environment) and a dedicated mobile phone. Should such a unit ever go into 
production the opportunities for reducing personal exposure to air pollution would be considerable.

\section{Conclusions}

The application of least-cost path analysis for defining routes which minimise journey-time exposure to air pollution has proved to be successful. Where alternative routes away from busy main roads can be found, reductions in exposure to $\mathrm{PM}_{10}$ of around $20 \%$ have been made relative to observed route data.

Creation of a detailed analysis mask is essential if results derived are to reflect realistic movements through space. There is potential to better reflect the realities of movement through space by incorporating other factors such as land surface type into the cost layer in order to represent the relative ease of movement across different surfaces.

It has also been noted, however, that many other factors influence route choice. For many individuals these other factors will affect the likelihood of alternative routes being adopted, especially where fear of particular spaces plays a significant role in route choice. In encouraging the use of less polluted routes, other influences on route choice cannot be ignored. Further work is, therefore, needed to assess the extent to which the varying concerns of different members of a population will affect the potential uptake of route alternatives developed.

\section{Acknowledgements}

We are grateful to the staff at the participating school and to the children involved for their commitment to the project and the rich data we have received from them. Thanks also to Marion Walker, Colin Pooley, Paul Coulton and William Bamford for their contributions to the wider project upon which the research presented here was based. The project was funded by the ESRC (Research Grant Small RES-000-22-2023). 


\section{References}

Arima E Y, Walker R T, Perz, S G and Caldas M. 2005. Loggers and forest fragmentation: Behavioural models of road building in the Amazon basin Annals of the Association of American Geographers 95:525-541.

Armstrong J M, and Khan A M. 2004. Modelling urban transportation emissions: role of GIS. Computers, Environment \& Urban Systems 28:421-433.

Atkinson D M, Deadman P, Dudycha D, and Traynor S. 2005. Multi-criteria evaluation and least cost path analysis for an arctic all-weather road. Applied Geography 25:287-307.

Bamford W, Coulton P, Walker M, Whyatt J D, Davies G, and Pooley C G. 2008. Using mobile phones to reveal the complexities of the school journey. In Proceedings of 10th International Conference on Human-Computer Interaction with Mobile Devices and Services. Accepted.

Beckett K P, Freer-Smith P, and Taylor, G. 2000. Effective tree species for local air-quality management. Journal of Arboriculture. 26:12-19

Borrego C, Tchepel O, Costa A M, Martins H, Ferreira J, and Miranda A I. 2006. Traffic-related particulate air pollution exposure in urban areas. Atmospheric Environment 40:7205-7214.

Briggs D. 2005. The Role of GIS: Coping with space (and time) in air pollution exposure assessment. Journal of Toxicology \& Environmental Health: Part A 68:1243-1261.

Briggs D J, de Hoogh K, Morris C, and Gulliver J. 2008. Effects of travel mode on exposure to particulate air pollution. Environment International 34:12-22.

CERC (Cambridge Environmental Research Consultants LTD) 2006. ADMS-Urban version 2.2. Cambridge, UK.

Chan L Y, Lau W L, Lee S C, and Chan C Y. 2002. Commuter exposure to particulate matter in public transportation modes in Hong Kong. Atmospheric Environment 36:3363-3373.

Collischonn W, and Pilar J V. 2000. A directional dependant least-cost-path algorithm for roads and canals. International Journal of Geographical Information Science 14:397-406.

Colvile R and D. J. Briggs D J. 2000. Dispersion modelling. In Elliott P, Wakefield J C, Best N G, and Briggs D J, editors. Spatial Epidemiology: Methods and Applications. Oxford University Press, New York: 375-392

Cook R, Isakov V, Touma J S, Benjey W, Thurman J, Kinnee E, and Ensley D. 2008. Resolving local-scale emissions for modelling air quality near roadways. Journal of the Air \& Waste Management Association 58:451-461.

Crabbe H, Hamilton R, and Machin N. 2000. Using GIS and dispersion modelling tools to assess the effect of the environment on health. Transactions in GIS 4:235-244.

DeMers M N. 2005. Fundamentals of Geographic Information Systems. Third edition. John Wiley and Sons, Inc.

DFT (Department for Transport) 2007. Travel in urban and rural areas. Personal Travel Fact Sheet July 2007.

Duncan M J, and Mummery W K. 2007. GIS or GPS? A comparison of two methods for assessing route taken during active transport. American Journal of Preventive Medicine 33:51-53.

Ellul C and Calnan B, 2008. Modelling street safety in an urban environment. In Proceedings of GIS Research UK 16th Annual Conference: 230-235.

English P, Neutra R, Scalf R, Sullivan M, Waller L, and Zhu L. 1999. Examining associations between childhood asthma and traffic flow using a geographic information system. Environmental Health Perspectives 107:761.

ESRI (Environmental Systems Research Institute Inc) 2006. ArcGIS Desktop 9.2. Redlands, California, USA.

Ganskopp D, Cruz R, and Johnson D E. 2000. Least-effort pathways?: a GIS analysis of livestock trails in rugged terrain. Applied Animal Behaviour Science 68:179-190.

Gauvin S, Reungoat P, Cassadou S, Dechenaux J, Momas I, Just J, and D. Zmirou. 2002. Contribution of indoor and outdoor environments to $\mathrm{PM}_{2.5}$ personal exposure of children - VESTA study. The Science of the Total Environment 297:178-181. 
Guardian Unlimited. 2007. Breathing Lessons http://www.guardian.co.uk/lifeandstyle/2007/apr/04/healthandwellbeing.waste. Accessed 13/08/08.

Gonzales E K and Gergel S E. 2007. Testing assumptions of cost surface analysis-a tool for invasive species management. Landscape Ecology 22:1155-1168.

Gordian M E, Haneuse S, and Wakefield J. 2006. An investigation of the association between traffic exposure and the diagnosis of asthma in children. Journal of Exposure Science \& Environmental Epidemiology 16:49-55.

Greaves S, Issarayandgyun T, and Lui Q. 2007. Exploring variability in pedestrian exposure to fine particulates (PM2.5) along a busy road. Atmospheric Environment 42:1665-1676.

Gulliver J and Briggs D J. 2004. Personal exposure to particulate air pollution in transport microenvironments. Atmospheric Environment 38:1-8.

Gulliver J and Briggs D J. 2005. Time-space modelling of journey-time exposure to traffic-related air pollution using GIS. Environmental Research 97:10-25.

Halpin P N and Bunn A G. 2000. Using GIS to compute a Least-Cost distance matrix: A Comparison of Terrestrial and Marine Ecological Applications. In Proceedings of ESRI Annual User Conference.

Harrison, R M, Deacon A R, Jones M R, and Appleby R S. 1997. Sources and processes affecting concentrations of $\mathrm{PM}_{10}$ and $\mathrm{PM}_{2.5}$ particulate matter in Birmingham (U.K.). Atmospheric Environment 31:4103-4117.

Heinrich J, Gehring U, Cyrys J, Brauer M, Hoek G, Fischer P, Bellander T, and Brunekreek. 2008. Exposure to traffic related air pollutants: self reported traffic intensity versus GIS modelled exposure. Occupational and Environmental Medicine 65:517-523

Heinrich J, Schwarze P E, Stilianakis N, Momas I, Medina S, Totlandsdal A I, von Bree L, Kuna-Dibbert B and Krzyzanowski M. 2005. Studies on health effects of transport-related air pollution. In Health effects of transport-related air pollution. Eds Krzyzanowski M, Kuna-Dibbert B and Schneider J. World Health Orgainsation. Denmark. pp125-165

Hertel O, Hvidberg M, Matthias Ketzel M, Storm L and Stausgaard L. 2008. A proper choice of route significantly reduces air pollution exposure - A study on bicycle and bus trips in urban streets. Science of the Total Environment 389:58-70.

Jensen S S, Berkowicz R, Sten Hansen H, and Hertel O. 2001. A Danish decision-support GIS tool for management of urban air quality and human exposures. Transportation Research Part D: Transport and Environment 6:229-241.

Jerrett M, Arain A, Kanaroglou P,Beckerman B, Potoglou D, Sahsuvaroglu T, Morrison J and Giovis C. 2005. A review and evaluation of intraurban air pollution exposure models. Journal of Exposure Analysis and Environmental Epidemiology.

Kaur S, Nieuwenhuijsen M J, and Colvile R. 2005. Pedestrian exposure to air pollution along a major road in Central London, UK. Atmospheric Environment 39:7307-7320.

Kaur S, Nieuwenhuijsen M J, and Colvile R N. 2007. Fine particulate matter and carbon monoxide exposure concentrations in urban street transport microenvironments. Atmospheric Environment 41:47814810.

Kearns R A, Collins D C A, and Neuwelt P M. 2003. The walking school bus: extending children's geographies? Area 35: 285-292.

Maynard D, Coull B A, Gryparis A, and Schwartz J. 2007. Mortality risk associated with short-term exposure to traffic particles and sulfates. Environmental Health Perspectives 115:751-755.

Michaels R A and Kleinman M T. 2000. Incidence and apparent health significance of brief airborne particle excursions. Aerosol Science \& Technology 32:93-105.

Nokia. 2007. Eco Sensor Concept. http://nokia.com/A4707477. Accessed 13/08/08.

Ordnance Survey (2007) OS MasterMap Topography Layer User Guide http://www.ordnancesurvey.co.uk/oswebsite/products/osmastermap/userguides/docs/OSMMTopoLa yerUserGuide.pdf Accessed 06/08

Osborne P. (2008) Safe routes to school and school travel plans. World Transport Policy and Practice. 14.1: 8-14

Regnauld N. and Mackaness W A. 2006. Creating a hydrographic network from its cartographic representation: a case study using Ordnance Survey MasterMap data. International Journal of Geographical Information Science. 60: 611-631 
RCEP (Royal Commission on Environmental Pollution). The urban environment. Report 26. http://www.rcep.org.uk/urban/report/urban-environment.pdf

Rothley K. 2005. Finding and filling the "cracks" in resistance surfaces for least-cost modelling. Ecology and Society 10. Article 4.

Walker M, Whyatt J D, Pooley C G, Davies G, Coulton P, and Bamford W. 2009. Talk, Technologies and Teenagers: Understanding the School Journey using a Mixed-Methods Approach. Children's Geographies In Press.

Whyatt J D, Davies G, Walker M, Pooley C G, Coulton P, and W Bamford W. 2008. Noisy school kids: Using GPS in an urban environment. In Proceedings of GIS Research UK 16th Annual Conference: 106-110.

Zandbergen P A and Green J W. 2007. Error and bias in determining exposure potential of children at school locations using proximity-based GIS techniques. Environmental Health Perspectives 115:1363-1370.

Zhao L, Wang X, He Q, Wang H, Sheng G, Chan L Y, Fu J, and Blake D R. 2004. Exposure to hazardous volatile organic compounds, $\mathrm{PM}_{10}$ and $\mathrm{CO}$ while walking along streets in urban Guangzhou. Atmospheric Environment 38:6177 - 6184. 